\title{
Streblid bat flies (Diptera) and other ectoparasites on bats (Mammalia: Chiroptera) from French Guiana
}

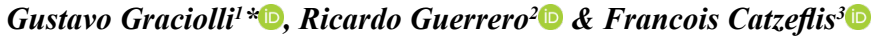 \\ ${ }^{1}$ Universidade Federal de Mato Grosso do Sul, Avenida Costa e Silva s/n., 79070-900, \\ Campo Grande, MS, Brasil \\ ${ }^{2}$ Universidad Central de Venezuela, Caracas, Distrito Capital, Venezuela \\ ${ }^{3}$ Institut des sciences de l'evolution, Faculté des Sciences, Montpellier, France \\ *Corresponding author: Gustavo Graciolli,e-mail: ggraciolli@yahoo.com.br
}

GRACIOLLI, G., GUERRERO, R., CATZEFLIS, F. Streblid bat flies (Diptera) and other ectoparasites on bats (Mammalia: Chiroptera) from French Guiana. Biota Neotropica. 19(4): e20180724. http://dx.doi.org/10.1590/1676-0611-BN-2018-0724

\begin{abstract}
Recent field surveys of bats (Chiroptera) in various localities of French Guiana have been accompanied by the collection and preservation of ectoparasites, mainly bat flies (Diptera: Streblidae and Nycteribiidae). Most specimens of ectoparasites was collected haphazardly during the course of bats inventories, but systematic surveys on the whole chiropteran community were realized at five opportunities. Concerning Streblidae, 813 individuals have been examined, which represent 46 species and/or subspecies belonging to 15 genera and 6 taxa for confirmation and/or future description. For Nycteribiidae, 44 individuals of 3 identified species and 2 for confirmation and/or future description. Other ectoparasites have been found (Hemiptera and acarids), which are also listed with details on their bat-host, place and date of collect. For six species of bats in which at least 10 animals were carrying ectoparasites, a brief description of the frequencies of their ectoparasites provides some preliminary characteristics of their infracommunities.
\end{abstract}

Keywords: Infracommunity, host-parasite relationship, Guianan bioregion.

\section{Moscas estréblidas (Diptera) e outros ectoparasitos de morcegos (Mammalia: Chiroptera) na Guiana Francesa}

Resumo: Pesquisas de campo recentes de morcegos (Chiroptera) em várias localidades da Guiana Francesa foram acompanhadas pela coleta e preservação de ectoparasitas, principalmente moscas ectoparasitas (Diptera: Streblidae e Nycteribiidae). A maioria dos espécimes de ectoparasitos foi coletada aleatoriamente durante o curso dos inventários de morcegos, mas pesquisas sistemáticas em toda a comunidade de quirópteros foram realizadas em cinco oportunidades. Sobre Streblidae, 813 indivíduos foram examinados, dos quais representam 46 espécies e/ou subespécies pertencentes a 15 gêneros e 6 táxons para posterior confirmação específica e/ou descrição. Para Nycterbiidae, 44 indivíduos de 3 espécies e 2 para posterior confirmação específica e/ou descrição. Outros ectoparasitos foram coletados (hemípteros polictenídeos e ácaros), os quais também foram listados com detalhes sobre seus hospedeiros, localidade e data de coleta. Para seis espécies de morcegos com mais de 10 morcegos infestados a descrição da composição de cada infracomunidade encontrada e sua frequência são apresentados.

Palavras-chave: Infracomunidade, relação hospedeiro-parasito, biorregião da Guiana. 


\section{Introduction}

Bats (Mammalia: Chiroptera) harbour a varied group of ectoparasitic arthropods, most of them occur exclusively on these mammalian hosts. Bat flies (Streblidae and Nycteribiidae), bat fleas (Ischnopsyllidae and one species of Hectopsylla [Tungidae]), bat bugs (Cimicidae and Polyctenidae), mites (Cheyletidae, Chirorhynchobiidae, Macronyssidae, Myobiidae, Spelaeorhynchidae, and Spinturnicidae), chiggers (Trombiculidae and Leeuwenhoekiidae) and tick (Argasidae and Ixodidae) are found parasiting bats in the Neotropical region (Whitaker et al. 2009). Important taxonomic surveys of ectoparasitic arthropods were carried out in Panama (Wenzel et al. 1966), Venezuela (Guimarães 1972, Jones et al. 1972, Ueshima 1972, Herrin \& Tipton 1975, Wenzel 1976, for example), Paraguay (Dick \& Gettinger 2005), Peru (Guerrero 1996-a, b), Argentina (see Autino et al. 2009, for example) and Brazil (see Graciolli et al. 2008). But in several areas of South America only occasional collections were made, resulting in a poor knowledge of the biodiversity of these ectoparasites and their host relationship. In French Guiana 12 species of streblid bat flies from six genera have been so far registered (Guerrero 1997).

On the hosts side, bats are rather common in all ecosystems of French Guiana, and several studies have described some aspects of their communities (Simmons \& Voss 1998, Charles-Dominique et al. 2001, Catzeflis et al. 2013).

Here we provide new data on streblid bat flies and their hosts and infracommunities, as per a combination of anecdotic and systematic collections carried out in French Guiana. We also provide data on nycteribiid bat flies, bat bugs (Hemiptera: Polyctenidae) and ticks and mites (Acari: Argasidae, Ixodidae, and Spinturnicidae) found on French Guianan bats.

\section{Material and Methods}

Ectoparasites were collected either haphazardly through the course of various bats inventories by experienced field naturalists or systematically during five surveys aimed at collecting Streblidae.

Altogether, the preserved ectoparasites come from 25 geographic localities spanning most of French Guiana (Figure 1).

Capture methods of Chiroptera included essentially mist nets set across nearby corridors such as trails in the forest. Mist nets of $2.6 \times$ $6 \mathrm{~m}$ and $2.6 \times 10 \mathrm{~m}(\mathrm{mesh}$ size $=16 \mathrm{~mm})$ were employed at ground level. In addition, we used at Cacao (locality 15 in Fig. 1) a three-frame harp trap (AUSTBAT Research Equipment, Victoria, Australia), with a catching surface of $1.0 \mathrm{~m} 2$ erected across a trail acting as a corridor for bats flying out of a nearby cave system.

Bats were handled individually in cloth bags, and first examined for the presence of ectoparasites, which were gently collected with the aid of tweezers and brushes through, and preserved in $70 \%$ ethanol.

Collected Diptera were taken to the laboratory and identified down to the specific level by Gustavo Graciolli and Ricardo Guerrero, with the aid of the following literature: Guimarães \& D'Andretta (1956), Guimarães (1966, 1972), Guerrero (1993, 1994, 1995a-b, 1996a). For Polyctenidae, Ixodidae and Spinturnicidae were consulted Ueshima (1972), Guerrero (1996c), Herrin \& Tipton (1975), respectively. For Acarids were mounted in Hoyer medium, and other taxa, when necessary, were examined in Lactophenol. The nomenclature of Streblidae and

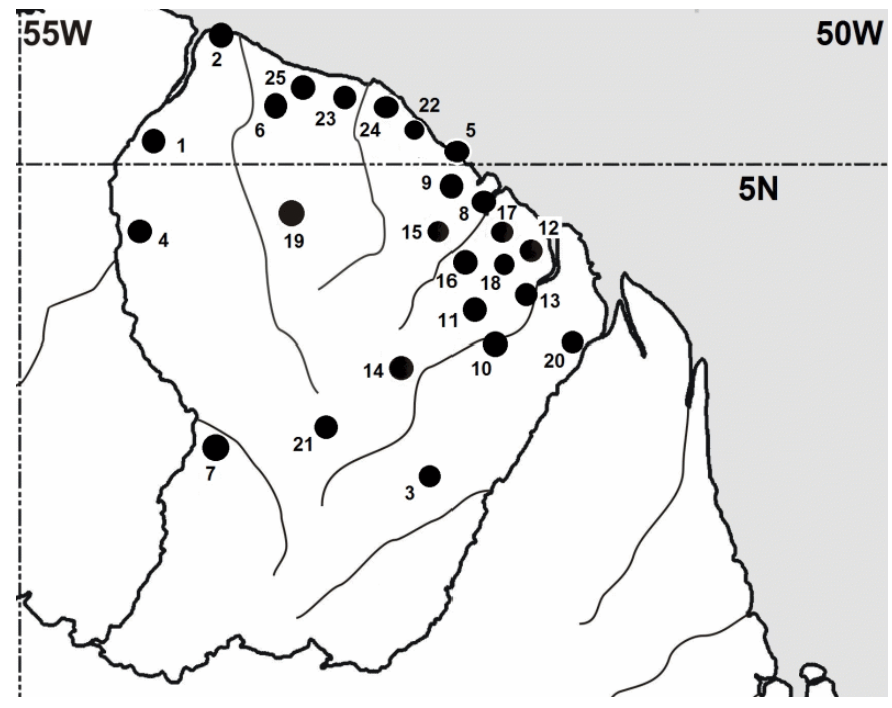

Figure 1. Map of French Guiana with the localities from where bats were caught with preserved ectoparasites. Localities are numbered 1 to 25 and correspond to: 1 = Apatou; 2 = Awala-Yalimapo; 3 = Camopi: Pic Coudreau du Sud; $4=$ Grand-Santi: Gaa Kaba (Montagne Francaise); $5=$ Kourou; $6=$ Mana: Crique Gargoulette along National Road; 7 = Maripasoula: Atachi Bakka; 8 = Matoury: Lagune Concorde; $9=$ Montsinery: Crique Cascade; $10=$ Regina: Approuague: Saut Athanase; 11 = Regina: Grande Montagne Tortue, piste de Belizon, pK13; 12 = Regina: Grotte Mathilde; 13 = Regina: Montagne des Gouffres; 14 = Regina: Nouragues; 15 = Roura: Cacao; 16 = Roura: Crique Boulanger; 17 = Roura: grotte Fourgassié; 18 = Roura: Trésor Nature Preservation; $19=$ Saint-Elie: Trinité Nature Preservation; 20 = Saint-Georges-Oyapock: TroisPalétuviers; 21 = Saül: near the village; $22=$ Sinnamary: Paracou at CIRAD; 23 = Sinnamary: Piste de Saint-Elie, pK-15; 24 = Sinnamary: National Road, pK-106; 25 = Sinnamary: Yiyi.

Nycteribiidae follows Guerrero (1997) and Graciolli et al. (2007), respectivelly. For Polyctenidae, Ixodidae and Spinturnicidae, we follow the proposal taxonomic showed in Ueshima (1972), Guerrero (1996c) and Herrin \& Tipton (1975).

Bat flies are deposited mainly at Coleção Zoológica de Referência da Universidade Federal de Mato Grosso do Sul, Campo Grande (Brazil), Colección de Parasitología del Museo de Ciencias de la Universidad Central de Venezuela (MBUCV) and also at Institut des Sciences de l'Evolution, Université de Montpellier (France). The other ectoparasites are deposited at MBUCV.

Identification of bats was done primarily based on Simmons \& Voss (1998) and on Charles-Dominique et al. (2001), but complementary literature and specimens for comparison were used as well. The nomenclature of bats follows Simmons (2005), with slight changes due to more recent taxonomical studies. Specifically, according to de Thoisy et al. (2014) and to Pavan et al. (2018), all Common Mustached Bats are identified either as Pteronotus rubiginosus (Wagner, 1843) emitting at $53 \mathrm{kHz}$ - or as Pteronotus alitonus Pavan, Bobrowiec \& Percequillo, 2018 - emitting at $59 \mathrm{kHz}$-. When no voucher has been preserved, and/or no molecular or bioacoustic character is available for a proper taxon identification, we name the Common Mustached Bats Pteronotus group-parnellii, as they were known in French Guiana before the study of de Thoisy et al. (2014). Following Velazco \& Patterson (2013; 2019), we name Sturnira giannae Velazco \& Patterson, 2019 all Little Yellow-shouldered Bats who were previously known as Sturnira lilium throughout the Guianan countries. 
External measurements (forearm, weight) were recorded for all captured bats after which they were released in the same spot. A small biopsy punch (Worthington \& Barratt 1996) used for DNA analyses was also taken from some individuals prior to release, and a small selection of specimens were preserved as scientific vouchers for further cranial measurements. These vouchered specimens were euthanized, fixed in $10 \%$ buffered formalin and finally stored in $70 \%$ ethanol. Appendix 1 lists the origin and institutions housing these vouchers. Bats were caught, handled, kept and euthanized following the guidelines of the American Society of Mammalogists for the use of wild mammals in research (Sikes et al. 2011). As no specific decree concerning Chiroptera outside protected areas exist in French Guiana, no specific legal authorization was required for captures and handling of bats. Vouchered bats were deposited in the collection of Museum d'Histoire Naturelle de Genève (MHNG).

The material examined is sorted by alphabetical order of species within subfamilies and families of ectoparasites. For each ectoparasite taxon, a few relevant comments are presented below, including at least the host species, locality and date of collect. Three species in the genus Trichobius (longipes group of taxa) appear new for science, and will be described in a separate paper.

For species of hosts with more than 10 infected individuals, we show the frequency of infracommunities found.

\section{Results}

During the five systematic surveys, 660 bats (of 56 species) were caught and examined for ectoparasites: 129 individuals (or 20\%) were found carrying bat flies. When considering only the eight species of bats caught each by at least 20 individuals, prevalence of bats harbouring ectoparasites ranges from zero (on two molossid species: Molossus coibensis and M. molossus) to 37\% (17 out of 46 Pteronotus alitonus), with an average of $13.5 \%$ (62 infested hosts out of 458 captured bats).

The ectoparasites listed here derive from 204 infested bats belonging to 37 species. Regarding hosts, we found ectoparasites on 75 (opportunistic) and 129 (systematic) individuals, which were from 24 (opportunistic) and 31 (systematic) species of bats.

\section{Insecta}

\section{DIPTERA}

Streblidae Kolenati, 1863

We examined 813 streblid bat flies of for a total of 46 species/subspecies and 15 genera and 6 taxa for confirmation and/or future description, of which 35 species/subspecies and 8 genera are registered for the first time in French Guiana.

Nycterophiliinae Wenzel, 1966

Nycterophilia coxata Ferris, 1916

Examined material: French Guiana: Regina: Nouragues, 1 male, ex Pteronotus group-parnellii, Maxime Cobigo leg., 6/III/2013.

Remarks: New geographic record for French Guiana.

Nycterophilia parnelli Wenzel, 1966

Examined material: French Guiana: Roura: Cacao, 1 male, ex Pteronotus group-parnellii, F. Catzeflis and M. Ruedi legs., 13/VII/2012.

Remarks: New geographic record for French Guiana.
Streblinae Speiser, 1900

Anastrebla caudiferae Wenzel, 1976

Examined material: French Guiana: Roura: Cacao, 1 male and 1 female, ex Anoura caudifer, Francois Catzeflis leg., 9/VII/2012.

Remarks: New geographic record for French Guiana.

Anastrebla modestini Wenzel, 1966.

Examined material: French Guiana: Cacao, Va-Joua, 1 male, ex Anoura geoffroyi, Benoit de Thoisy leg., 11/VI/2011.

Remarks: New geographic record for French Guiana.

Anastrebla spurrelli Wenzel, 1976

Examined material: French Guiana: Saint-Elie: Trinité Nature Preservation, 1 female, ex Lionycteris spurrelli, M. Delaval and V. Ruffray leg., 10/IX/2011; same locality, 1 male, same host and collectors but 12/IX/2011.

Remarks: New geographic record for French Guiana.

Metelasmus pseudopterus Coquillett, 1907

Examined material: French Guiana: Regina: Nouragues, 1 female, ex Artibeus planirostris, M. Cobigo leg., 14/VIII/2013. Roura: Cacao, 1 male, same host, Francois Catzeflis leg., 10/VII/2012.

Remarks: New geographic record for French Guiana.

Strebla alvarezi Wenzel, 1966

Examined material: French Guiana: Roura: Cacao, 1 male, ex Micronycteris megalotis, Francois Catzeflis leg., 11/VII/2012. SaintElié: Trinité Nature Preservation, 1 female, same host, J. F. Cosson leg., 12/IX/1991.

Remarks: Previously quoted by Guerrero (1997).

\section{Strebla christinae Wenzel, 1966}

Examined material: French Guiana: Regina: Grande Montagne Tortue, piste de Belizon, pK-13, 2 males, ex Phylloderma stenops, Margot Delaval leg., 13/X/2010. Maripasoula, Atachi Bakka, 13 males and 7 females, ex 2 P. stenops, Sylvain Uriot leg., IX/2011.

Remarks: New geographic record for French Guiana.

Strebla consocia Wenzel, 1966

Examined material: French Guiana: Saint-Elie: Trinité Nature Preservation, 1 female, ex Phyllostomus elongatus, M. Delaval and V. Ruffray leg., 6/IX/2011; same locality, 1 male, same host and collectors but 12/IX/2011; same locality, 1 male and 1 female, same host and collectors but 14/IX/2011; same locality, 1 male, ex Phyllostomus hastatus, M. Delaval and V. Ruffray leg., 10/IX/2011; same locality, 1 female, same host and collectors but 11/IX/2011; same locality, 5 males and 5 females, ex 3 P. hastatus, same collectors but 15/IX/2011. Maripasoula: Atachi Bakka, 3 males and 4 females ex 2 P. hastatus, Sylvain Uriot leg., IX/2011. Sinnamary: Paracou at CIRAD-camp, 1 male, ex Artibeus obscurus, Francois Catzeflis leg., 10/X/2013. Sinnamary: Piste de Saint-Elie, pK-15, 1 female, ex P. hastatus, J.-F. Cosson leg., 23/I/1990; same locality, 1 male and 1 female, ex Pteronotus rubiginosus, J.-F. Cosson leg., 8/XI/1991. Awala-Yalimapo, 1 male, ex P. hastatus, M. Delaval leg., 29/X/2011. Roura: Cacao, 1 male and 2 females, same host, 14/VII/2012.

Remarks: Previously quoted by Guerrero (1997). 
Strebla diaemi Wenzel, 1966

Examined material: French Guiana: Regina: Approuague, Saut Athanase, 2 males and 3 females, ex 2 Diaemus youngi, Benoit de Thoisy leg., 18/VIII/2012.

Remarks: New geographic record for French Guiana.

Strebla guajiro (García \& Casal, 1965)

Examined material: French Guiana: Roura: Cacao, 3 females, ex 2 Carollia perspicillata, Francois Catzeflis leg.,19/VI/2011; same locality, 1 female, same host and collector but 21/VI/2011; same locality, 4 females, ex 2 C. perspicillata, same collector, 7/VII/2012; same locality, 5 males and 2 females, same host and collector but 9/VII/2012; same locality, 5 males and 2 females, same hosts and collector but 9/ VII/2012; same locality, 1 male and 1 female, same host and collector but 12/VII/2012; same locality, 1 female, same host, Benoit de Thoisy leg., 11/XI/2011. Sinnamary: Piste de Saint-Elie, pK-15, 1 male, same host, J.-F. Cosson leg., 1990.

Remarks: Previously quoted by Guerrero (1997).

Strebla harderi Wenzel, 1976

Examined material: French Guiana: Roura: Cacao, 2 males, ex Lonchophylla thomasi, Francois Catzeflis leg., 9/VII/2012.

Remarks: New geographic record for French Guiana.

Strebla hertigi Wenzel, 1966

Examined material: French Guiana: Sinnamary: Piste de Saint-Elie, pK-15, 1 male, ex Phyllostomus elongatus, J.-F. Cosson leg., 1/VI/1990. Roura: Cacao, 2 males, ex Phyllostomus discolor, Francois Catzeflis leg., 14/VII/2012.

Remarks: Previously quoted by Guerrero (1997).

Strebla mirabilis (Waterhouse, 1879)

Examined material: French Guiana: Roura: grotte de Fourgassier, Route de Kaw, 1 male and 1 female, ex Trachops cirrhosus, Benoit de Thoisy leg., 12/VI/2011. Saint-Elié: Trinité Nature Preservation, 1 male, same host, M. Delaval and V. Ruffray legs., 10/IX/2011.

Remarks: Previously quoted by Guerrero (1997).

Strebla obtusa Wenzel, 1976

Examined material: French Guiana: Apatou: 1 male and 1 female, ex Trinycteris nicefori, Margot Delaval leg., 04/09/2010. Roura, Cacao: 3 males and 1 female, ex T. nicefori, Francois Catzeflis leg., 9/VII/2012; same locality, 7 males and 1 female, same host and collector but 21/ $\mathrm{VII} / 2012$.

Remarks: New geographic record for French Guiana.

Strebla wiedemanni Kolenati, 1856

Examined material: French Guiana: Regina: Nouragues, Arataye, 2 males, ex Desmodus rotundus, Margot Delaval leg., 30/X/2010. Roura: grotte de Fourgassier (Route de Kaw), 6 males and 3 females, ex 3 D. rotundus, Benoit de Thoisy leg., 12/VI/2011. Saut-Athanase, Fleuve Approuague, 2 males, ex D. rotundus, Benoit de Thoisy leg., 26/VI/2011; same locality, 2 males and 1 male, same host, M. Delaval leg., 20/XI/2011. Saint-Elie, Trinité Nature Preservation, 2 males and 2 females, same host, M. Delaval and V. Ruffray legs., 14/IX/2011. Regina, grotte Mathilde, 4 males and 4 females, same host, M. Delaval leg., 27/I/2013. Regina, Approuague, Saut Athanase, 2 males, same host, Benoit de Thoisy leg., 26/VI/2011

Remarks: New geographic record for French Guiana.

Strebla $\mathrm{cf}$. paramirabilis

Examined material: French Guiana: Roura: Cacao, 1 female, ex Mimon bennetti, Francois Catzeflis leg., 12/VII/2012.

Strebla sp.

Examined material: French Guiana: Roura: Cacao, 1 female, ex Lonchophylla thomasi, Francois Catzeflis, 13/VII/2012.

Trichobiinae Jobling, 1936

Aspidoptera falcata Wenzel, 1976

Examined material: French Guiana: Roura: Cacao, 2 males and 2 females, ex Sturnira giannae, Francois Catzeflis leg., 19/VI/2011, same locality, 9 males and 8 females, same host and same collector, 21/VI/2011; same locality, 1 male and 1 female, same host, F. Catzeflis and M. Ruedi legs., 6/VII/2012; same locality, 3 females, same hosts and collectors but $9 / \mathrm{VII} / 2012$.

Remarks: New geographic record for French Guiana. This represents a new host record. Aspidoptera falcata is often found on bats of the genus Sturnira (Guerrero 1995b).

Aspidoptera phyllostomatis (Perty, 1833)

Examined material: French Guiana: Roura: Cacao, 1 male and 2 females, ex 3 Artibeus planirostris, Francois Catzeflis leg., 10/VII/2012; same locality, 1 male, same host and collector but 21/VI/2011.

Regina, Nouragues, 1 male and 4 females, same host, M. Cobigo leg., 14/VIII/2013.

Remarks: New geographic record for French Guiana.

Mastoptera minuta (Costa Lima, 1921)

Examined material: French Guiana: Saint-Elie, Trinité Nature Preservation, 1 male, ex Lophostoma sylvaticum, M. Delaval and V. Ruffray legs., 14/IX/2011; same locality, 4 males and 7 females, ex Phyllostomus hastatus, same collectors, 10/IX/2011; same locality, 1 male and 2 females, same host, same collectors but 15/IX/2011. Roura: Cacao, 3 males and 3 females, same host, F. Catzeflis and M. Ruedi legs., 10/VII/2012.

Remarks: New geographic record for French Guiana.

Megistopoda aranea (Coquillett, 1899)

Examined material: French Guiana: Roura: Cacao, 1 male and 1 female, ex 2 Artibeus planirostris, F. Catzeflis leg., 10/VII/2012.

Remarks: New geographic record for French Guiana.

Megistopoda proxima (Séguy, 1926)

Examined material: French Guiana: Roura: Cacao, 1 male, ex Sturnira giannae, F. Catzeflis leg., 19/VI/2011; same locality, 4 males ex 3 Sturnira giannae, same collector but 21/VI/2011; same locality, 1 male and 1 female, same host, Catzeflis and M. Ruedi legs., 9/VII/2012; same locality, 1 male, ex Sturnira tildae, same collectors, 12/VII/2012; same locality, 1 male, Sturnira giannae, Francois Catzeflis leg., 23/VII/2012. Kourou, 1 female, ex Sturnira sp., J.-F. Cosson leg., no data. 
Remarks: Previously quoted by Guerrero (1997). This represents a new host record. Megistopoda proxima is often found on bats of the genus Sturnira (Guerrero 1995b).

Neotrichobius bisetosus Wenzel, 1976

Examined material: French Guiana: Saint-Elie, Trinité Nature Preservation, 1 male, ex Artibeus planirostris, M. Delaval and V. Ruffray legs., 14/IX/2011. Regina, Nouragues, 2 males and 1 female, same host, M. Cobigo leg., 14/VIII/2013.

Remarks: New geographic record for French Guiana.

Neotrichobius delicatus (Machado-Allison, 1966)

Examined material: French Guiana: Roura: Cacao, 1 male, ex Rhinophylla pumilio, F. Catzeflis leg., 11/VI/2011; 1 male and 2 females, ex 2 R. pumilio, F. Catzeflis and M. Ruedi legs., 7/VII/ 2012. Saint-Elie: Trinité Nature Preservation, 1 female, same host, M. Delaval and V. Ruffray legs., 12/IX/2011.

Remarks: New geographic record for French Guiana.

Neotrichobius delicatus complex

Examined material: French Guiana: Roura: Cacao, 1 male, ex Dermanura gnoma, F. Catzeflis leg., 26/VI/2011.

Neotrichobius stenopterus Wenzel \& Aitken, 1966

Examined material: French Guiana: Roura: Cacao, 1 male, ex Pteronotus group-parnelli, F. Catzeflis and M. Ruedi legs., 13/VII/2012; same locality, 1 female, ex Dermanura gnoma, Francois Catzeflis leg., 18/ VII/2012.

Remarks: New geographic record for French Guiana.

Noctiliostrebla aitkeni Wenzel, 1966

Examined material: French Guiana: Awala-Yalimapo, 4 males and 6 females, ex 3 Noctilio leporinus, M. Delaval leg., 30/X/2011.

Remarks: New geographic record for French Guiana. Recently Alcantara et al. (2019) published a taxonomic revision of Noctiliostrebla, restricting the records of $N$. aitkeni for Brazil, Ecuador, Trinidad and Tobago and Venezuela.

Noctiliostrebla maai Wenzel, 1966

Examined material: French Guiana: Awala-Yalimapo, 4 males and 5 females, ex 4 Noctilio albiventris, M. Delaval leg., 30/X/2011.

Remarks: New geographic record for French Guiana. Previously this species was recorded in Colombia, Panama and Venezuela (Alcantara et al. 2019).

\section{Paradyschiria lineata Kessel, 1925}

Examined material: French Guiana: Awala-Yalimapo, 7 males and 6 females, ex 3 Noctilio leporinus, M. Delaval leg., 30/X/2011. Matoury: Lagune Concorde, 2 males and 1 female, same host and collector, 4/ XI/2011. Sinnamary: Yiyi, 2 females, same host, J.-F. Cosson leg. Remarks: New geographic record for French Guiana.

Paradyschiria parvula Falcoz, 1931

Examined material: French Guiana: Awala-Yalimapo, 18 males and 16 females, ex 7 Noctilio albiventris, M. Delaval leg., 31/X/2011.

Remarks: New geographic record for French Guiana.
Parastrebla handleyi Wenzel, 1966

Examined material: French Guiana: Apatou, Trinycteris nicefori, 3 males, Margot Delaval leg., 04/09/2010. Roura: Cacao, 1 female, same host, Francois Catzeflis leg., 21/VII/2012.

Remarks: New geographic record for French Guiana.

\section{Pseudostrebla riberoi Costa Lima, 1921}

Examined material: French Guiana: Sinnamary: Piste de Saint-Elie, pK15, 1 female, ex Micronycteris megalotis, J.-F. Cosson leg., 13/I/1990. Remarks: Previously quoted by Guerrero (1997) as Pseudostrebla microtis litt. error, a nomem nudum.

Speiseria ambigua Kessel, 1925

Examined material: French Guiana: Sinnamary: Piste de Saint-Elie pK15, 1 female, ex Carollia perspicillata, 1990. Roura: Cacao: Va-Joua, ex C. perspicillata, 3 males and 3 females, ex 4 C. perspicillata, Benoit de Thoisy leg., 11/VI/2011; same locality, 1 male, same host, F. Catzeflis leg., 19/VI/2011; same locality, 1 male and 2 females, same host and collector but 21/VI/2011. Maripasoula: Atachi Bakka, 1 female, ex Trachops cirrhosus, Sylvain Uriot leg., 1/IX/2011. Saint-Elie: Trinité Nature Preservation, 1 male, same host, M. Delaval and V. Ruffray legs., 10/IX/2011. Roura: Cacao, 1 male, ex Trinycteris nicefori, F. Catzeflis leg., 9/VII/2012; same locality, 1 male and 1 female, ex C. perspicillata, same collector and date; same locality, 1 female, same host and collector but7/VII/2012. Saint Georges-Oyapock: Trois Paletuviers, 2 females, same host and collector, 5/VII/2013.

Remarks: Previously quoted by Guerrero (1997).

Speiseria magnioculus Wenzel, 1976

Examined material: French Guiana: Maripasoula: Atachi Bakka, 1 female, ex Trachops cirrhosus, Sylvain Uriot leg., 1/IX/ 2011.

Remarks: New geographic record for French Guiana.

Trichobioides perspicillatus (Pessôa and Galvão, 1937)

Examined material: French Guiana: Roura: Cacao, 1 male and 1 female, ex Phyllostomus discolor, Francois Catzeflist leg., 14/VII/2012. Remarks: New geographic record for French Guiana.

Trichobius anducei Guerrero, 1998

Examined material: French Guiana: Roura: Cacao: Va-Joua, 8 males and 4 females, ex 4 Carollia perspicillata, Benoit de Thoisy leg., 11/ VI/2011; same locality, 4 males and 6 females, ex 2 C. perspicillata, Francois Catzeflis leg., 19/VI/2011; same locality, 3 males and 1 female, same host and collector, 21/VI/2011; same locality, 4 females, ex $2 C$. perspicillata, same collector, 7/VII/2012; same locality, 1 male, same host and collector but 9/VII/2012.

Remarks: New geographic record for French Guiana.

\section{Trichobius caecus Edwards, 1918}

Examined material: French Guiana: Sinnamary: Piste de Saint-Elie, pK-15, 4 males, 1 female, ex Pteronotus rubiginosus, J.-F. Cosson leg., 8/XI/1991. Grand-Santi: Gaa Kaba (Montagne Francaise), 1 male and 3 females, same host, M. Delaval leg., 22/IX/2010. Regina: Grande Montagne Tortue, piste de Belizon, pK13, 3 males, same host, M. Delaval leg., 15/X/2010. Regina: Grotte Mathildae, 1 male, 1 female, ex P. rubiginosus, K. Pineau and M. Delaval legs., 2011; same locality, 
2 males and 2 females, ex 2 Pteronotus group-parnelli, same collectors and date. Roura: Trésor Nature Preservation, 3 males and 3 females ex 3 Pteronotus group-parnelli, K. Pineau and M. Delaval legs., 2011; same locality, 1 female, ex $P$. rubiginosus, same collectors and date. Saint-Elie: Trinité Nature Preservation, 6 males and 10 females, ex 2 Pteronotus alitonus, M. Delaval and V. Ruffray legs., 9/IX/2011; same locality, 25 males and 13 females, ex 4 Pteronotus alitonus, same collectors, 10/IX/2011; same locality, 7 males and 3 females, ex Natalus tumidirostris, same collectors, 12/IX/2011; same locality, 2 males and 2 females, ex Pteronotus alitonus, same collectors, 14/IX/2011; same locality, 8 males and 3 females, same host and collectors, 15/IX/2011. Awala-Yalimapo, 1 male, ex Noctilio albiventris, M. Delaval leg., 31/X/2011. Regina: Montagne des Gouffres, 2 males and 2 females, M. Delaval leg., 3 males and 1 female, ex Pteronotus alitonus, same collector, 22/XI/2011; same locality, 1 male and 1 female, ex $P$. rubiginosus, same collector and date. Roura: Cacao, 2 males and 2 females, ex Pteronotus alitonus, F. Catzeflis and M. Ruedi legs., 9/ VII/2012; same locality, 3 males, ex 2 Pteronotus alitonus, same collectors, 11/VII/2012; same locality, 1 female, same host and collector, 12/VII/2012; same locality, 8 males and 13 females, ex 4 P. rubiginosus, same collectors, 13/VII/2012; same locality, 9 males and 15 females, 4 ex Pteronotus alitonus, same collectors and date; same locality, 6 males and 4 females, same host and collectors, 14/VII/2012; same locality, 1 male and 1 female, ex P. rubiginosus, same collectors and date; same locality, 17 males and 18 females, same host and collectors, 19/VII/2012. Regina: Nouragues, 6 males and 3 females ex P. rubiginosus, Maxime Cobigo leg., 12/X/2012; same locality, 4 males and 3 females, same host and collector, 6/III/2013; same locality, 11 males and 5 females, same host and collector, 12/VIII/2013.

Remarks: In French Guiana, Guerrero (1997) had already recorded this species on Pteronotus group-parnelli without information about locality. The occurrence of $T$. caecus on $P$. alitonus represents a new host record. Other species of Pteronotus parasitized by T. caecus are P. davyi and P. parnellii (Guerrero 1994).

Trichobius diaemi Wenzel, 1976

Examined material: French Guiana: Regina. Approuague: Saut Athanase. 10 males and 6 females, ex 2 Diaemus youngi, Benoit de Thoisy, 18/VIII/2012.

Remarks: New geographic record for French Guiana.

Trichobius dugesioides dugesioides Wenzel, 1966

Examined material: French Guiana: Saul: near the village, 1 female, ex Carollia perspicillata, J.-F. Cosson, leg., 18/VII/1990. Regina: Grande Montagne Tortue, piste de Belizon, pK-13, 2 females, Trachops cirrhosus, Margot Delaval leg., 16/X/2010. Roura: grotte de Fourgassier (Route de Kaw), 2 females, same host, Benoit de Thoisy leg., 12/ VI/2011. Maripasoula: Atachi Bakka, 1 male and 5 females, ex 3 T. cirrhosus, Sylvain Uriot leg., IX/2011. Saint-Elie: Trinité Nature Preservation, 1 female, same host, M. Delaval and V. Ruffray legs., 10/IX/2011. Roura: Cacao, 1 male and 2 females, ex Mimon bennettii, Francois Catzeflis leg., 14/VII/2012.

Remarks: In French Guiana, Guerrero (1997) had already recorded this species on Carollia perspicillata from Saül.
Trichobius dugesioides phyllostomus Guerrero, 1998

Examined material: French Guiana: Regina: Grande Montagne Tortue, piste de Belizon, pK-13, 1 male, ex Phyllostomus elongatus, Margot Delaval leg., 13/X/2010. Maripasoula: Atachi Bakka, 7 males and 3 females, same host, Sylvain Uriot leg., IX/2011. Saint-Elie: Trinité Nature Preservation, 1 male and 1 female, same host, M. Delaval and V. Ruffray leg. 6/IX/2011; Saint-Elie: Trinité Nature Preservation, 1 male and 1 female, same host, M. Delaval and V. Ruffray leg. 12/IX/2011; Saint-Elie: Trinité Nature Preservation, 1 male and 3 females, same host, M. Delaval and V. Ruffray leg. 14/IX/2011.

Remarks: New geographic record for French Guiana.

\section{Trichobius joblingi Wenzel, 1966}

Examined material: French Guiana: Sinnamary: Piste de Saint-Elie, pK15, 2 males and 3 females, ex Carollia perspicillata, J.-F. Cosson leg., 1990. Maripasoula: Atachi Bakka, 1 male and 1 female, ex Trachops cirrhosus, Sylvain Uriot leg., IX/2011. Roura: Cacao: Va-Joua, C. perspicillata, 1 female, Francois Catzeflis leg., 19/VI/2011; same locality, 1 female, same collector, 7/VII/2012; same locality, 2 males and 4 females, ex 2 C. perspicillata, Francois Catzeflis leg., 9/VII/2012; same locality, 3 males and 3 females, same host and collector but 12/ VII/012; same locality, 2 males, ex Micronycteris megalotis, Francois Catzeflis leg., 11/VII/2012. Saint Georges-Oyapock: Trois-Paletuviers, 3 females, ex Carollia perspicillata, Francois Catzeflis leg., 5/VII/2013. Remarks: In French Guiana, Guerrero (1997) had already recorded this species on Carollia perspicillata without information about locality.

\section{Trichobius jonhsonae Wenzel, 1966}

Examined material: French Guiana: Roura: Cacao: Va-Joua, 1 male and 1 female, Pteronotus rubiginosus, Francois Catzeflis leg., 11/VI/2011; same locality, 11 males and 24 females, Pteronotus personatus, F. Catzeflis \& M. Ruedi legs., 23/VII/2012; same locality 2 males and 1 female, same host and collectors, 25/VII/2012; same locality 9 males and 3 females, same host and collectors, 27/VII/2012.

Remarks: New geographic record for French Guiana.

\section{Trichobius keenani Wenzel, 1966}

Examined material: French Guiana: Roura: Cacao, 1 male, ex Micronycteris megalotis, Francois Catzeflis leg., 11/ VII/2012.

Remarks: New geographic record for French Guiana.

Trichobius lionycteridis Wenzel, 1966

Examined material: French Guiana: Saint-Elie: Trinité Nature Preservation, 1 male and 1 female, ex 2 Lionycteris spurrelli, M. Delaval and V. Ruffray legs., 10/IX/2011; same locality, 2 males and 1 female, ex 2 L. spurrelli, same collectors, 12/IX/2011; same locality, 6 males and 3 females, ex 3 L. spurrelli, same collectors, 15/IX/2011. Roura: Cacao, 1 male and 1 female, same host, F. Catzeflis leg., 10/VII/2012. Remarks: New geographic record for French Guiana.

Trichobius longipes (Rudow, 1871)

Examined material: French Guiana: Roura: Cacao, 4 males, ex Phyllostomus hastatus, F. Catzeflis and M. Ruedi legs., 14/VII/2012. Maripasoula: Atachi Bakka, 1 male, ex Phyllostomus elongatus, Sylvain 
Uriot leg., IX/2011. Saint-Elie: Trinité Nature Preservation, 2 males and 2 females, ex $P$. hastatus, M. Delaval and V. Ruffray legs., 9/IX/2011; same locality, 1 male and 2 females, same host and collectors, 10/ IX/2011; same locality, 2 males and 5 females, ex 3 P. hastatus, same collectors, 15/IX/2011: Awala-Yalimapo, 1 female, same host, M. Delaval leg., 28/X2011.

Remarks: New geographic record for French Guiana.

\section{Trichobius parasiticus Gervais, 1844}

Examined material: French Guiana: Regina: Grande Montagne Tortue, piste de Belizon, pK-13, 1 male, ex Desmodus rotundus, Margot Delaval leg., 14/X/2010. Roura: grotte de Fourgassier (Route de Kaw), 9 males and 5 females, ex 4 D. rotundus, Benoit de Thoisy leg., 12/VI/2012; same locality, 3 males and 4 females, same host, M. Delaval leg., 20/ XI/2011. Régina: Saut-Athanase, Fleuve Approuague, D. rotundus, 1 male, Benoit de Thoisy leg., 26/VI/2011. Regina: Approuague: Saut Athanase, 1 male, same host and collector but 26/VI/2011. Saint-Elie: Trinité Nature Preservation, 1 female, same host, M. Delaval and V. Ruffray legs., 14/IX/2011.

Remarks: New geographic record for French Guiana.

\section{Trichobius sparsus Kessel, 1925}

Examined material: French Guiana: Saint-Elie: Trinité Nature Preservation, 2 males, ex Pteronotus alitonus, M. Delaval and V. Ruffray legs., 10/IX/2011; same locality, 1 female, same host and collectors but 14/IX/2011; 1 female, same host and collectors but 15/IX/2011.

Remarks: New geographic record for French Guiana.

\section{Trichobius tiptoni Wenzel, 1976}

Examined material: French Guiana: Sinnamary Piste de Saint-Elie pK15, 2 males, ex Anoura caudifer, J.-F. Cosson, 1990.

Remarks: Previously quoted by Guerrero (1997).

\section{Trichobius sp. (parasiticus complex)}

Examined material: French Guiana: Cacao: Va-Joua, ex Micronycteris sp., 1 male and 2 females, Benoit de Thoisy leg., 11/VI/2011.

Trichobius sp. n. 1 (longipes group)

Examined material: French Guiana: Mana: Crique Gargoulette along National Road, 2 males and 1 female, ex Molossus rufus, M. Delaval leg., 2/XI/2011.

\section{Trichobius sp. n. 2 (longipes group)}

Examined material: French Guiana: Roura: Cacao, 3 males and 1 female, ex Lonchophylla thomasi, F. Catzeflis leg., 9/VII/2012.

Trichobius sp. n. 3 (longipes group)

Examined material: French Guiana: Sinnamary: Paracou at CIRAD camp, 3 males, 1 female, ex Artibeus obscurus, F. Catzeflis leg., $10 / \mathrm{X} / 2013$.
Nycteribiidae Samouelle, 1819

Basilia carteri Scott, 1936

Examined material: French Guiana: Montsinery: Crique Cascade, ex. Myotis albescens, 2 males and 6 females, M. Dewynter leg., 31/ VII/2011. Regina: Grotte Mathilde, 1 female, ex Myotis cf riparius, M. Delaval leg., 2/VII/2011; same locality, 1 male and 1 female, ex Myotis nigricans, Francois Catzeflis leg., 25/VII/2012; same locality, 1 male and 1 female, ex Myotis sp., same collector and date. AwalaYalimapo, 1 male, ex Myotis sp., same collector, 29/X/2011. Roura: Crique Boulanger, 1 female, same host and collector, 7/XI/2011. Sinnamary: National Road, pK-106, 3 males and 4 females, ex 3 Myotis sp., F. Catzeflis leg., 1/X/2013; same locality, 1 female, same host and collector but $7 / \mathrm{X} / 2013$.

Remarks: New geographic record for French Guiana.

Basilia ortizi Machado-Allison, 1963

Examined material: French Guiana: Awala-Yalimapo, 6 males and 2 females, ex Eptesicus furinalis, M. Delaval leg., 31/X/2011.

Remarks: New geographic record for French Guiana.

Basilia sp.

Examined material: French Guiana: Sinnamary: Yiyi, 2 females, ex Eptesicus furinalis, M. Delaval leg., 3/XI/2011.

Remarks: This specimen could be an undescribed species belongs to ferruginea group.

Basilia travassosi Guimarães, 1938

Examined material: French Guiana: Awala-Yalimapo, 2 males and 4 females, ex 3 Myotis sp., M. Delaval leg., 29/X/2011.

Remarks: New geographic record for French Guiana. Previously, this species was restricted Northeastern of Brazil (Ceará, Maranhão, Paraíba, and Pernambuco states) on species of Myotis (Graciolli et al., 2007; Santos et al., 2013; Barbier et al. 2016; Bezerra \& Bocchiglieri, 2018; Barbier et al., 2019). This extending the distribution of $B$. travassosi more than $1,500 \mathrm{~km}$ (distance between Awala-Yalimapo and Bairrerinhas, Maranhão).

Basila cf. mimoni Theodor \& Peterson, 1964

Examined material: French Guiana: Camopi: Pic Coudreau du Sud, 1 male and 4 females, ex Mimon crenulatum, M. Delaval leg., 4/II/2013.

\section{HEMIPTERA}

Polyctenidae Westwood, 1874

Hesperoctenes cartus Jordan, 1922

Examined material: French Guiana: Saint-Elie: Trinité Nature Preservation, 1 male and 1 female, ex Cynomops paranus, M. Delaval and V. Ruffray legs., 10/IX/2011. Mana: Crique Gargoulette along National Road, 1 female, ex Cynomops abrasus, M. Delaval leg., 2/ $\mathrm{XI} / 2011$. Sinnamary: Yiyi, 1 female, same host and collector, 3/XI/2011; same locality, 2 males and 6 females, ex 3 Cynomops planirostris, same collector and date.

Remarks: New geographic record for French Guiana. 
Hesperoctenes fumarius (Westwood, 1874)

Examined material: French Guiana: Saint-Elie: Trinité Nature Preservation, 1 female, ex Molossus rufus, M. Delaval and V. Ruffray legs., 14/IX/2011. Mana: Crique Gargoulette along National Road, 1 male, ex Cynomops abrasus, M. Delaval leg., 2/XI/2011; 1 male, 1 female and 2 nymphs, ex 2 M. rufus, same collector but 1/XI/2011. Remarks: New geographic record for French Guiana.

\section{Acarina}

\section{IXODOIDEA}

Argasidae Canestrini 1890

Ornithodoros hasei (Schulze, 1935)

Examined material: French Guiana: Awala-Yalimapo, 3 larvae, ex 2 Noctilio leporinus, M. Delaval leg., 30/X/2011; same locality, 6 larvae, ex Noctilio albiventris, same collector, 31//2011.

Remarks: New geographic record for French Guiana.

Ornithodoros marinkellei Kohls, Clifford \& Jones, 1969

Examined material: French Guiana: Roura: Cacao, 1 larva, ex Ptenotus rubiginosus, F. Catzeflis and M. Ruedi legs., 13/VII/2012.

Remarks: New geographic record for French Guiana.

Ornithodoros sp.

Examined material: French Guiana: Saint-Elie: Trinité Nature Preservation, 1 larva, ex Micronycteris megalotis, J.-F. Cosson leg., 12/ IX/1991. Same locality, 2 larvae, ex 2 Pteronotus alitonus, M. Delaval and V. Ruffray legs., 9/IX/2011. Roura: Trésor Nature Preservation, 1 larva, ex Pteronotus rubiginosus, K. Pineau and M. Delaval legs., 2011.

\section{DERMANYSSOIDEA}

Spinturnicidae Oudemans, 1901

Spinturnix americanus Banks, 1902

Examined material: French Guiana: Montsinery: Crique Cascade, 1 female, ex Myotis albescens, M. Dewynter leg., 31/VII/2011. Roura: Cacao, 1 female, ex Myotis nigricans, F. Catzeflis leg., 25/VII/2012. Sinnamary: National Road, pK106, 1 male and 1 protonymph, ex Myotis sp., same collector, $1 / \mathrm{X} / 2013$.

Remarks: New geographic record for French Guiana.

Spinturnix surinamensis Dusbabek and Lukoschus, 1971

Examined material: French Guiana: Sinnamary: Yiyi, 1 female, ex Eptesicus furinalis, M. Delaval leg., 3/XI/2011.

Remarks: New geographic record for French Guiana.

Periglischrus micronycteridis Furman, 1966

Examined material: French Guiana: Saint-Elie: Trinité Nature Preservation, 1 female, ex Micronycteris megalotis, J.-F. Cosson leg., 12/IX/1991. Roura: Cacao, 1 male, ex Trinycteris nicefori, F. Catzeflis leg., 21/VII/2012.

Remarks: New geographic record for French Guiana.
Periglischrus paravargasi Herrin and Tipton, 1975

Examined material: French Guiana: Saint-Elie: Trinité Nature Preservation, 6 females, ex Anoura caudifer, J.-F. Cosson leg., 20/V/1992.

Remarks: New geographic record for French Guiana.

Spelaeorhynchidae Oudmans, 1902

Spelaeorhynchus sp.

Examined material: French Guiana: Regina: Grotte Mathilde, 16 females, ex 4 Pteronotus rubiginosus, K. Pineau and M. Delaval legs., 2011.

\section{Discussion}

Previously Guerrero (1997) cited and registered 12 species of 6 genera from French Guiana, most of which originating from Piste de Saint-Elie (locality number 23 on Figure 1). Adding our results, the number of species of streblid bat flies increases to 58 (Table 1). But the number of streblid bat flies should be much higher, if we consider the 102 species of bat recorded in French Guiana (Lim \& Tavares 2012). In neighboring countries that harbour a high richness of bats there has also been recorded higher richness of streblid bat flies. For example in Brazil there are 178 species of bats (Nogueira et al. 2014) and 83 species of streblid bat flies (Graciolli 2018), in Venezuela 166 bats (Gardner 2007) and 121 bat flies (Guerrero 1997) and in Colombia 180 species of bats (Gardner 2007) and 54 species of Streblidae (Marinkelle \& Grose 1981; Dick et al. 2016), respectively.

We show the infracommunities found on six species of host with a sample size of at least 10 infested individuals. For Carollia perspicillata, we found nine infracommunities with one to three species. The most frequent infracommunities have two species. Few information is available for infracommunities composition and frequency on bats' ectoparasites. Santos et al. (2013), considering only infracommunities with two or more species, found three infracommunities with two and three species on $C$. perspicillata in Maranhão state, Brazil. There $T$. jobling $i$ was the species that occurred in all infracommunities, whereas in French Guiana, T. joblingi and T. anducei were found in four out of nine infracommunities. These two Trichobius species were observed together in only one infracommunity.

Regarding the species of the mormoopid genus Pteronotus, we note that $P$. rubiginosus showed the most infracommunities with one species (16 out of 17 infracommunities); to the contrary, on the species $P$. alitonus, the majority of the infracommunties was composed of two species. On both species of mustached bats, the most frequent infracommunity was composed only by Trichobius caecus.

On Desmodus rotundus and Sturnira giannae were found the same number of infracommunities with same numbers of species. But on $D$. rotundus, the most frequent infracommunity had two species, whereas on $S$. giannae, infracommuties were composed by only one species (Aspidoptera falcata). Komeno \& Linhares (1999) showed a negative correlation between A. falcata and M. proxima on Sturnira lilium and this could be an evidence of competition between these species. The 
Table 1. Species of bat host with more than 10 parasitized specimens (number of specimens between brackets) and infracommunities of ectoparasitic arthropods frequencies and relative frequencies.

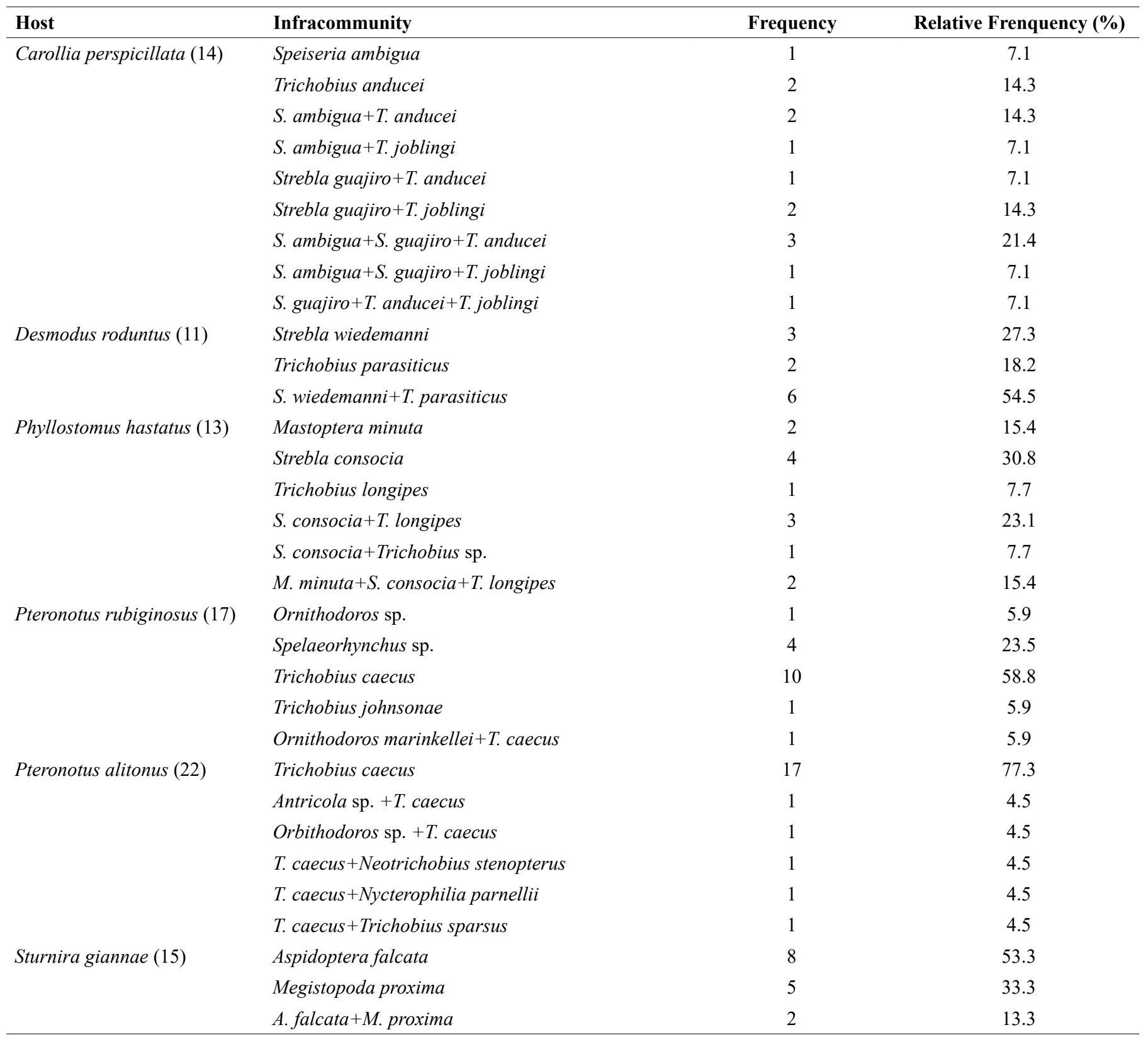

infracommunities characteristics (richness and composition) could be influenced by several factors such as host availability, competition including host available, geographic area, bat flies species composition, competition with other ectoparasites, or else roost quality (Wenzel et al. 1966). Furthermore, along the geographic range of a bat species, the various populations can harbor different infracommunities.

\section{Conclusion}

Despite the new field surveys bringing 41 additional species to the Streblidae fauna of French Guiana, it appears that the number of streblid bat flies and other species of bat 's ectoparasites remains less than expected based onto comparisons with neighboring countries.
Clearly, new field work in various environments, savannahs, marshes, terra firme forests, mountains, of the whole of French Guiana are needed for a better understanding of the biodiversity, the distribution and the host relationship of arthropods ectoparasites of bats in this part of the Guianan Shield.

\section{Acknowledgements}

GG thanks to CNPq (process \# 302328/2012-2) and FUNDECT $(23 / 200.638 / 2014 ; 23 / 200.662 / 2014)$ for grant support. FC thanks all French Guianan collectors of bats who kindly preserved ectoparasites at our request: Maxime Cobigo, Jean-François Cosson, Marguerite Delaval, Benoit de Thoisy, Maël Dewynter, Kevin Pineau, Vincent 
Ruffray, and Sylvain Uriot. Manuel Ruedi spent considerable time with us at Cacao for sampling several hundreds of bats, and generously offering the opportunity of brushing those for ectoparasites. Bats caught in Nature Preservation localities (Trésor; Trinité) with due permission of administrative authorities. This work has benefited from an "Investissements d'Avenir" grant managed by the French Agence Nationale de la Recherche (CEBA, ref. ANR-10-LABX-25-01).

\section{Author contributions}

Gustavo Graciolli: Contribution to data analysis and interpretation; Contribution to manuscript preparation; Contribution to critical revision, adding intelectual content.

Ricardo Guerrero: Contribution to data analysis and interpretation; Contribution to manuscript preparation; Contribution to critical revision, adding intelectual content.

Francois Catzeflis: Substantial contribution in the concept and design of the study; Contribution to manuscript preparation; Contribution to critical revision, adding intelectual content.

\section{Conflicts of interest}

The authors declare that they have no conflict of interest related to the publication of this manuscript.

\section{References}

ALCANTARA, D.M.C., GRACIOLLI, G. \& NIHEI, S.S. 2019. Revision of Noctiliostrebla (Diptera: Streblidae), parasites of bulldog bats (Chiroptera: Noctilionidae: Noctilio). Zootaxa 4560: 483-521.

AUTINO, A.G., CLAPS, G.L., SÁNCHEZ, M.S. \& BARQUEZ, R.M. 2009. New records of bat ectoparasites (Diptera, Hemiptera and Siphonaptera) from Northern Argentina. Neotrop Entomol 38: 165-177.

BARBIER, E., PRADO-NETO, J.G. \& BERNARD, E. 2016. Records of bat flies (Diptera: Nycteribiidae and Streblidae) in the semi-arid Caatinga in the state of Paraíba, Northeastern Brazil. Neotrop Entomol 45: 740-745.

BARBIER, E., GRACIOLLI, G. \& BERNARD, E. 2019. Structure and composition of Nycteribiidae and Streblidae flies on bats along an environmental gradient in northeastern Brazil. Can. J. Zool. 97: 409-418.

BEZERRA, R.H.S. \& BOCCHIGLIERI, A. 2018. Association of ectoparasites (Diptera and Acari) on bats (Mammalia) in a restinga habitat in northeastern Brazil. Parasitol Res 117: 3413-3420.

CATZEFLIS, F., DEWYNTER, M. \& PINEAU, K. 2013. Liste taxonomique commentée des chiroptères de Guyane. Le Rhinolophe 19: 89-102.

CHARLES-DOMINIQUE, P., BROSSET, A. \& JOUARD, S. 2001. Les chauvessouris de Guyane. Patrimoines Naturels 49: 1-150.

DE THOISY, B., PAVAN, A.C., DELAVAL, M., LAVERGNE, A., LUGLIA, T., PINEAU, K., RUEDI, M., RUFRAY, V. \& CATZEFLIS, F. 2014. Cryptic diversity in common mustached bats Pteronotus cf. parnellii (Mormoopidae) in French Guiana and Brazilian Amapá. Acta Chiropterol. 16: 1-13.

DICK, C.W. \& GETTINGER, D. 2005. A faunal survey of streblid flies (Diptera: Streblidae) associated with bats in Paraguay. J. Parasit. 91: 1015-1024.

DICK, C.W., GRACIOLLI, G. \& GUERRERO, R. 2016. Family Streblidae [Catalogue of Streblidae of Colombia]. Zootaxa 4122: 784-802.

GARDNER, S.L. 2007. Mammals of South America: Marsupials, Xenarthrans, Shrews, and Bats. V. 1. University of Chicago, Chicago, p. 690.

GRACIOLLI, G. 2018. Streblidae in Catálogo Taxonômico da Fauna do Brasil. PNUD. (http://fauna.jbrj.gov.br/fauna/faunadobrasil/2624). Access on 05 Jul. 2018.
GRACIOLLI, G., AUTINO, A.G. \& CLAPS, G.L. 2007. Catalogue of American Nycteribiidae (Diptera, Hippoboscoidea). Rev. Bras. Entomol. 51: 142-159.

GRACIOLLI, G., AZEVEDO, A.A., ARZUA, M., BARROS-BATTESTI, D.M. \& LINARDI, P.M. 2008. Artrópodos Ectoparasitos de Morcegos no Brasil. In Morcegos no Brasil: Biologia, sistemática, ecologia e conservação (S.M. Pacheco, R.V. Marques \& C.E.L. Esbérard, eds). 1 edição. Armazém Digital, Porto Alegre, p. 123-138.

GUERRERO, R. 1993. Catálogo de los Streblidae (Diptera: Pupipa) parásitos de murciélagos (Mammalia: Chiroptera) del Nuevo Mundo. 1. Clave para los géneros y Nycterophiliinae. Acta Biol. Venez. 4: 61-75.

GUERRERO, R. 1994. Catálogo de los Streblidae (Diptera: Pupipa) parásitos de murciélagos (Mammalia: Chiroptera) del Nuevo Mundo. IV. Trichobiinae con alas desarrolladas. Bol. Entomol. Venez., N. S. 9: 161-191.

GUERRERO, R. 1995a. Catálogo de los Streblidae (Diptera: Pupipara) parásitos de murciélagos (Mammalia: Chiroptera) del Nuevo Mundo. III. Los grupos: dugesii, dunni, y phyllostomae del Género Trichobius Gervais, 1844. Acta Biol. Venez. 15: 1-27.

GUERRERO, R. 1995b. Catálogo de los Streblidae (Diptera: Pupipa) parásitos de murciélagos (Mammalia: Chiroptera) del Nuevo Mundo. V. Trichobiinae con alas reducidas o ausentes y miscelaneo. Bol. Entomol. Venez., N. S. 10: $135-160$.

GUERRERO, R. 1996a. Catálogo de los Streblidae (Diptera: Pupípara) parasitos de murciélagos (Mammalia: Chiroptera) del nuevo mundo VI. Streblinae. Acta Biol. Venez. 16: 1-25.

GUERRERO, R. 1996b. Streblidae (Diptera: Pupipara) parásitos de los murciélagos de Pakitza, Parque Nacional Manu (Perú). In MANU: La biodiversidad del sureste del Perú (D.E. Wilson \& A. Sandoval eds). Washington, DC, Smithsonian Institution, p. 627-641.

GUERRERO, R. 1996c. Garrapatas de Venezuela (Acarina: Ixodoidea). Listado de especies y claves para su identificación. Bol. Dir. Malariol. Saneam. Ambient. 36: 1-24.

GUERRERO, R. 1997. Catálogo de los Streblidae (Diptera: Pupipara) parásitos de murciélagos (Mammalia: Chiroptera) del Nuevo Mundo. VII. Lista de especies, hospedadores y paises. Acta Biol. Venez. 17: 9-24.

GUIMARÃES, L.R. 1966. Nycteribiid batflies from Panama. In Ectoparasites of Panama (R.L. Wenzel \& V.J. Tipton, eds). Field Museum of Natural History, Chicago, Illinois, p. 393-404.

GUIMARÃES, L.R. 1972. Venezuelan nycteribiid batflies (Diptera: Nycteribiidae). Brigham Young Univ. Sci. bull. Biol. Ser. 17(1): Article 1. Available at: https://scholarsarchive.byu.edu/byuscib/vol17/iss1/1.

GUIMARÃES, L.R. \& D'ANDRETTA, M.A.V. 1956. Sinopse dos Nycteribiidae (Diptera) do Novo Mundo. Arq. Zool. Est. São Paulo 10:1-184.

HERRIN, C.S. \& TIPTON, V.J. 1975. Spinturnicid mites of Venezuela (Acarina: Spinturnicidae). Brigham Young Univ. Sci. bull. Biol. Ser. 20(2): Article 1. Available at: https://scholarsarchive.byu.edu/byuscib/vol20/iss2/1

JONES, E.K., CLIFFORD, C.M., KEIRANS, J.E. \& KOHLS, G.M. 1972. Ticks of Venezuela (Acarina: Ixodoidea), with a key to the species of Amblyomma in the Western Hemisphere. Brigham Young Univ. Sci. bull. Biol. Ser. 17(4): Article 1. Available at: https://scholarsarchive.byu.edu/byuscib/vol17/iss4/1.

KOMENO, C.A. \& LINHARES, A.X. 1999. Batflies parasitic on some phyllostomid bats in Southeasthern Brazil: parasitism rates and host-parasite relationships. Mem. Inst. Oswaldo Cruz 94: 151-156.

LIM, B.K. \& TAVARES, V. da C. 2012. Review of species richness and biogeography of bats (Chiroptera) from the Guiana subregion of South America, with comments on conservation. Ecotropica 18: 105-118.

MARINKELLE, C.J. \& GROSE, E.S. 1981. A list of ectoparasites of Colombian bats. Rev. Biol. Trop. 29: 11-20.

PAVAN, A.C., BOBROWIEC, P.E.D. \& PERCEQUILLO, A.R. 2018. Geographic variation in a South American clade of mormoopid bats, Pteronotus (Phyllodia), with description of a new species. J. Mammal. 99:624-645

NOGUEIRA, M.R., de LIMA, I.P., MORATELLI, R. \& TAVARES, V. da C. 2014. Checklist of Brazilian bats, with comments on original records. Check List 10: 808-821. 
SANTOS, C.L.C., PEREIRA, A.C.N., BASTOS, V. de J.C., GRACIOLLI, G. \& RÊBELO, J.M.M. 2013. Parasitism of ectoparasitic flies on bats in the northern Brazilian cerrado. Acta Parasitol. 58: 207-214.

SIKES, R.S., GANNON, W.L. \& THE ANIMAL CARE AND USE COMMITTEE OF THE AMERICAN SOCIETY OF MAMMALOGISTS. 2011. Guidelines of the American Society of Mammalogists for the use of wild mammals in research. J. Mammal. 92:235-253.

SIMMONS, N.B. 2005. Order Chiroptera. In Mammal species of the World - A taxonomic and geographic reference (D.E. Wilson \& D.M. Reeder, D.M. eds). Third edition. The Johns Hopkins University Press, Baltimore, p. 312-529.

SIMMONS, N.B. \& VOSS, R.S. 1998. The mammals of Paracou, French Guiana: A Neotropical lowland rainforest fauna. Part 1. Bats. Bull. Am. Mus. Nat. Hist. 237: 1-219.

UESHIMA, N. 1972. New World Polyctenidae (Hemiptera), with special reference to Venezuelan species. Brigham Young Univ. Sci. bull. Biol. Ser. 17(1): Article 2. Available at: https://scholarsarchive.byu.edu/byuscib/ vol17/iss $1 / 2$.
VELAZCO, P.M. \& PATTERSON, B.D. 2013. Diversification of the Yellowshouldered bats, Genus Sturnira (Chiroptera, Phyllostomidae), in the New World tropics. Mol. phylogenet. Evol. 68: 683-698.

VELAZCO, P. M. \& PATTERSON, B.D. 2019. Small mammals of the Mayo River Basin in northern Peru, with the description of a new species of Sturnira (Chiroptera, Phyllostomidae). Bull. Am. Mus. Nat. Hist. 429:1-67.

WENZEL, R.L. 1976. The streblid batflies of Venezuela (Diptera: Streblidae). Brigham Young Univ. Sci. bull. Biol. Ser. 20(4): Article 1. Available at: https://scholarsarchive.byu.edu/byuscib/vol20/iss4/1.

WENZEL, R.L. \& TIPTON, V.J. 1966. Ectoparasites of Panama. Field Museum of Natural History, Chicago, Illinois.

WENZEL, R.L., TIPTON, V.J. \& KIEWLICZ, A.1966. The Streblid bat flies of Panamá (Diptera: Calypterae: Streblidae). In Ectoparasites of Panama (R.L. Wenzel \& V.J. Tipton, eds). Field Museum of Natural History, Chicago, Illinois, p. 405-675.

WHITAKER, Jr., J.O., RITZI, C.M. \& DICK, C.W. 2009. Collecting and preserving bat ectoparasites for ecological study. In Ecological and behavioral methods for the study of bats (T.H. Kunz \& S. Parsons, eds). John Hopkins University Press, Baltimore, p. 806-827.

WORTHINGTON, W.J. \& BARRATT, E.M. 1996. A non-lethal method of tissue sampling for genetic studies of chiropterans. Bat Research News 37: 1-3.

Received: 18/01/2019

Revised: 09/07/2019

Accepted: 12/07/2019

Published online: 15/08/2019 